\title{
Adapting Single-User Visualization Software for Collaborative Use
}

\author{
Francis T. Marchese, Jude Mercado, and Yi Pan \\ Department of Computer Science \\ Pace University \\ New York, NY 10038 \\ fmarchese@pace.edu
}

\begin{abstract}
This paper presents our experiences with adapting single-user visualization software for web-based collaboration. Sun's Java JXTA API was used to adapt an opensource molecular visualization program called Jmol. It was found that by focusing on the program's graphical user interface the software could be quickly transformed into a peer-to-peer application. Our positive experience implies that many useful single-user programs should be transformable into tools that make collaboration across the web easier to initiate, more spontaneous, and supported by a wide range of visualization software.
\end{abstract}

\section{Introduction}

The Internet has made it possible for individuals or groups to collaborate at a distance. Collaborative virtual environments (CVEs) have been developed to support a wide range of collaborative activities including gaming, immersive virtual reality systems for military training, scientific visualization, and engineering design [1]. Collaborative visualization software systems include VisAD [2], COVISA [3], Sieve [4], and others [5-7]. In general, these programs have been developed to provide a generic set of collaborative tools for data sharing, representation, and visualization. However, many domain-specific applications do not easily adapt to a more general class of visualization programs. Yet there are many single-user programs that not only would be useful collaborative tools but also could be adapted for collaborative use. Finally, many collaborative systems require dedicated hardware and software, even though many collaborations may be infrequent, of short duration, take place at odd hours, or employ an amalgam of mainstream conferencing and problem-specific software products.

Therefore, we decided to investigate these issues by adapting a domain-specific, single-user program for collaborative use. The goal was to create web-based software that did not rely on special hardware and could be used at any time, in any place. We selected an open-source mo- lecular visualization program written in Java called Jmol [8] and Sun's Java peer-to-peer (P2P) toolkit - JXTA [9 11]. When the project began, we had little experience with peer-to-peer systems and had never seen Jmol's source code. Some of our experiences are presented herein.

The following section contains the background for the project. Section 3 contains a discussion of peer-to-peer computing with JXTA. Section 4 presents the process of adapting Jmol for collaborative use, and Section 5 a sample session for running Jmol in peer-to-peer collaboration. Discussion and conclusions are found in Section 6. Section 7 makes suggestions for future work.

\section{Background}

Molecular visualization is a traditional example of a domain-specific visualization application. Molecular visualization environments tightly integrate data generation, visualization, and analysis. Moreover, they rely on graphical representations not found in main stream visualization systems. The process of displaying electron densities, molecular vibrations, and protein structures has evolved since the early days of computer graphics in the mid-1960s. Today, individual or small clusters of singleuser, PC-based molecular visualization systems can compute and render molecular structure and dynamics in near real-time.

A number of collaborative molecular visualization systems have been built, including PaulingWorld [12], Chimera [13], and Mice [14]. PaulingWorld is a virtual reality application designed to work on SGI workstations with immersive hardware systems such as CAVEs, Collaborative Workbenches, and head mounted displays. Alternatively, MICE (Molecular Interactive Collaborative Environment) is a web-based, client-server system built on Java3D and CORBA. Users translate molecular structure data into VRML geometry files and load them onto the server. These files are then collaboratively viewed through Java clients. Finally, Chimera is a client-server collaborative system that wraps a collection of legacy 
molecular analysis and visualization programs within a GUI.

These systems illustrate a number of issues related to collaborative systems. First, many CVEs require special purpose hardware and software. This is the case with PaulingWorld, which runs only on SGI computers attached to VR hardware. Second, CVEs may require nonnative data models. For example, Mice transforms molecular structure into VRML objects comprised of geometric primitives such as polygon meshes and surface patches. This loss of original geometry means there is no way to quantitatively interrogate the source data; thus leaving collaborators with only the ability to rotate, translate, or scale objects. Finally, although many CVEs allow users to directly manipulate source data, they are clientserver applications. Chimera allows users access to the original molecular data, but like Mice, it is a client-server application, and as such, its collaborative use is subject to constraints. Since the server is responsible for data transmission, it must have a resolvable IP address for clients to make a connection. The Internet's growth has left fewer machines with static IP addresses, resulting in powerful computing systems assigned dynamic IP addresses that may inhibit their abilities to be used as servers. And client-server applications cannot traverse firewalls. Firewall technology is designed to protect private information. However, there is a need for users to communicate through a firewall in order to perform collaborative research.

Hence, collaborative molecular visualization software should support direct access to source data and metadata, manipulation of native visual representations, interactive data query and update, and anytime-anywhere collaboration. One possible way to create such an application is to begin with a pre-existing single user visualization system and adapt it for P2P use. Because molecular scientists typically recreate their software with each new generation of technology, there are many molecular visualization applets written in Java, used to embed molecular visualizations within web pages $[8,15-16]$. One such program is Jmol [8].

JMol is an open-source molecular visualization program. Chemical scientists use Jmol as a visualization and measurement tool. It is capable of animating the results of simulations and can perform transformations (rotation, translation, scaling) upon a molecular structure. In addition, the application can also measure inter-atomic distances, bond angles, and dihedral angles, as well as print and export captured images. A snapshot of a Jmol session is displayed in Figure 1.

The advantage of using a Java-based program is that it can be paired with JXTA, Sun's Java peer-to-peer protocol that allows any network-connected device to communicate and collaborate. Peer-to-peer networking solves client-server problems by interconnecting machines so

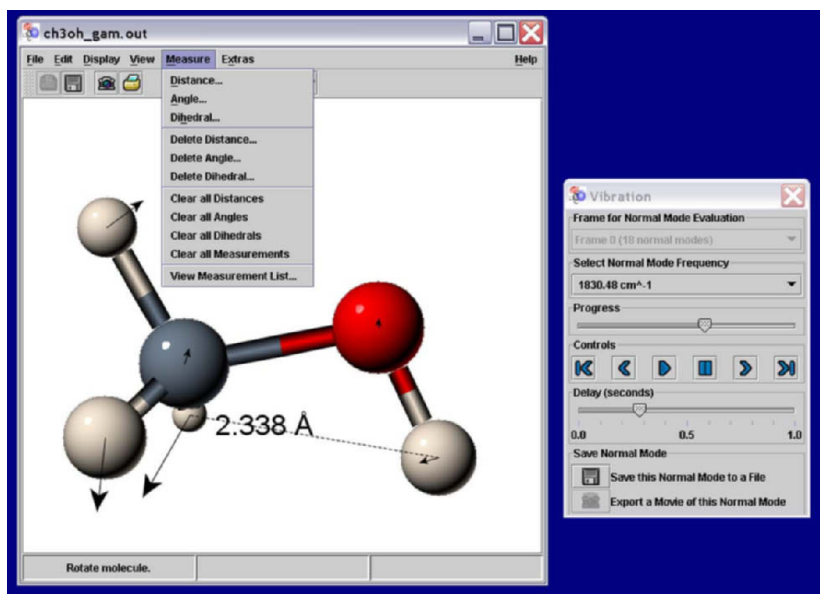

Figure 1 Jmol GUI showing a molecular representation with VCR controls for displaying molecular dynamics

that each node acts as both server and client. There is no single center in a P2P network as there is in a clientserver network. P2P services are distributed, promoting the robustness of the entire network by eliminating the static IP address requirement for data transmission. In addition, with multiple protocols to select for P2P applications and certain types of peers in use, P2P application users can communicate through a firewall.

\section{JXTA Background}

JXTA is a P2P networking framework, which consists of three layers: Platform, Services, and Applications. Plaform is the core layer with the elements for every P2P solution. Services provide the access to JXTA protocols. Applications use services to access the JXTA network and utilities.

JXTA's goal is to support P2P programming on any device from a PDA to a toaster regardless of where it may be located. Conceptually, it is designed to organize peers and provide an infrastructure for communication. JXTA does this with protocols expressed in XML.

JXTA is founded on the following basic concepts: peer, peer group, endpoint, pipe, network transport, advertisements, protocols, and discoveries.

A peer is a virtual communication point. There are three basic types of peers: simple peer, rendezvous peer, and router peer. A simple peer has the least functionality, and is used behind a firewall. A rendezvous peer processes queries from other peers, and is used when content transmission is required. A router peer enables peers to communicate with other peers separated by a firewall.

A peer group organizes peers, and provides specific services to group members. Data may be shared within the group's scope, and peers may check another peer's status 
before it is allowed to join the group, subject to security requirements.

Network transport manages data transmission over the network. JXTA allows peers to choose different protocols to fit specific needs. HTTP protocols are used for peers to communicate through firewalls, while TCP is chosen for intranets.

A network transport system is composed of endpoints, pipes, and messages. An endpoint refers to an address of a peer. A pipe is a unidirectional, asynchronous, and virtual connection of two or more endpoints. Messages contain the data being transmitted. Transmitted data is packed into a message, which is then sent over the output pipe. At the other end of the pipe, a peer receives the message from its input pipe and extracts the transmitted data.

An advertisement is a structured representation of an entity, service, or resource made available by a peer or peer group as a part of a P2P network. It is an XML document in JXTA, containing the description of a message, peer, peer group, or service. Peers discover advertisements on the network to find other peers. They can use a cached advertisement, rendezvous peer, or router peer to discover each other within a LAN or through a firewall.

JXTA has a number of protocols for advertising, sending, routing, propagating, and securing messages. These protocols are used to join a peer group, find another peer, create pipes between peers, and propagate messages among peers. These protocols are used to effect peer-topeer collaboration.

A typical scenario for P2P collaboration using JXTA is as follows:

\section{Start JXTA}

2. Create and publish pipe advertisements

3. Locate and use the created pipe advertisement

4. Send outgoing and listen for incoming messages.

An application initiates collaboration by joining the Net peer group. The default Net group gives access to the basic JXTA services. When the JXTA platform is started, it searches for certain configuration files, most importantly the PlatformConfig file. PlatformConfig is an XML file that records network settings specific to the peer. If this file is not present a JXTA Configuration screen is shown (Figure 2). It is here that the username, password and advanced network specifications can be configured.

A Pipe Advertisement is created using the AdvertisementFactory class. The Pipe Advertisement is an XML file that contains the type of pipe, the Pipe ID, and possibly an optional name for the pipe. Once created, peers bind to the pipe's input and output endpoints to create instances of the InputPipe and OutputPipe class.

\section{Implementation}

Jmol is an event-driven system with its graphical user interface (GUI) controlling most events. Jmol's GUI is based on Java's Swing interface toolkit (JDK 1.2 and

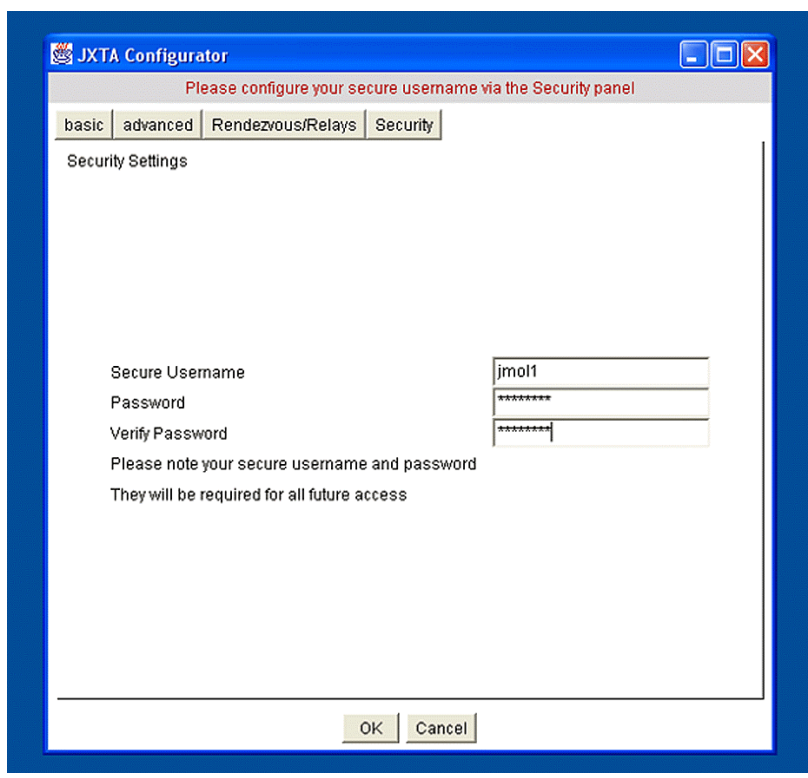

\section{Figure 2 Configuration dialog for JXTA applica- tion}

later), a refined version of Java's Abstract Window Toolkit (AWT) found in Java's JDK 1.1 and later. Each interface component, or widget, is linked to a listener interface that captures a unique event. Thus, when the mouse is moved, or a button is pushed, or a dropdown menu item is selected, an event occurs that is captured and acted upon. And Java's listener interface provides specific methods in which to embed actions to be taken once an event has been captured.

By finding the Java methods in Jmol that control the listener interface it should be possible to insert the JXTA code necessary to transmit an event and its attributes to a peer. Jmol's interface events result in loading and saving files, updating the molecular graphic representation, and generating geometric data to describe inter-atomic distances and angles. After the first peer acts on the event by updating its local state, it should send a unique message to the second peer for it to replicate the process. For example, the sending peer captures combined button and mouse events for translating the molecular representation, and redraws it at a new screen location. After translation, a message is sent to the receiving peer, corresponding to a translation action. The receiving peer then updates its state to match the sending peer.

In addition to updating Jmol's event handling methods, three other issues needed to be resolved in order to transform Jmol into a collaborative system. First, a database model needed to be selected. A distributed database model was assumed in which each site retains a copy of all files used in Jmol. The advantage is that file replication reduces transmission times and creates minimal communication time lag, since only changes in the pro- 


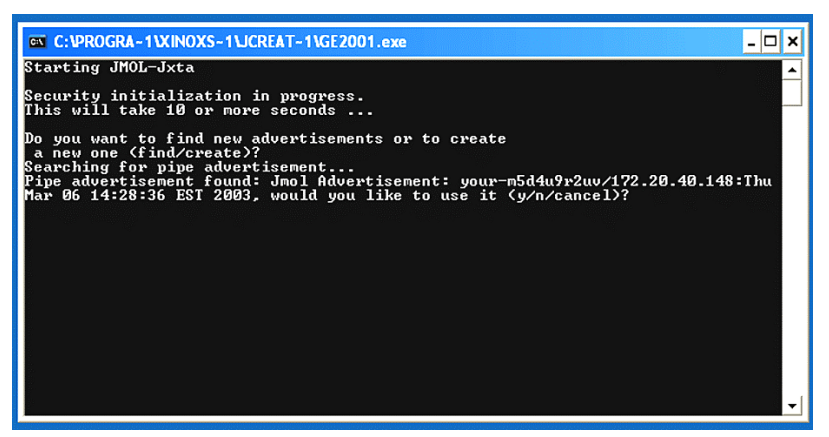

\section{Figure 3 Collaborative Jmol searching for peer advertisement.}

gram's state need be transmitted. The logic behind this is that most molecular data can be easily downloaded or transmitted, and collaborative users would already have these on their machines.

Second, JXTA code must be added to implement the essential JXTA protocols. In particular, communication channels must be established, incoming messages handled, pipe advertisements discovered, and output pipes created.

Finally, messages between peers should be compact in order to minimize communication overhead. For Jmol, messages only need to include a key specifying an action and an element containing the attributes of that action. For example, a translation operation would send the key "XLATE" along with an element containing the translation matrix. The receiving peer, upon recognizing the message, would use the transformation matrix to perform a translation locally.

With strategy in hand, Jmol was adapted for collaboration by adding code to set up the pipes necessary for communication channels among peers and modifying the graphical user interface (GUI) components. Upon analysis, it was found that out of the 270 class files that constitute Jmol, the GUI components are isolated in five files: DisplayPanel.java, Animate.java, Vibrate.java, and Measure.java.

DisplayPanel.java contains controls for geometric transformations as well as rendering options. There are action methods for picking, rotation, zooming, translation, shading, and the like. Every "Action" method found within DisplayPanel was modified to send a message to a listening peer for it to perform the same task.

Animate.java creates the Animate class, which controls the animation of reaction simulations. It displays VCR controls that allow the user to start, stop, rewind, forward, jump to a frame, or speed up the simulation. When a user chooses to 'Play,' 'Rewind,' 'Fast Forward,' etc., a message corresponding to the action is sent to the listening peer so that it can change the frame on its local application.

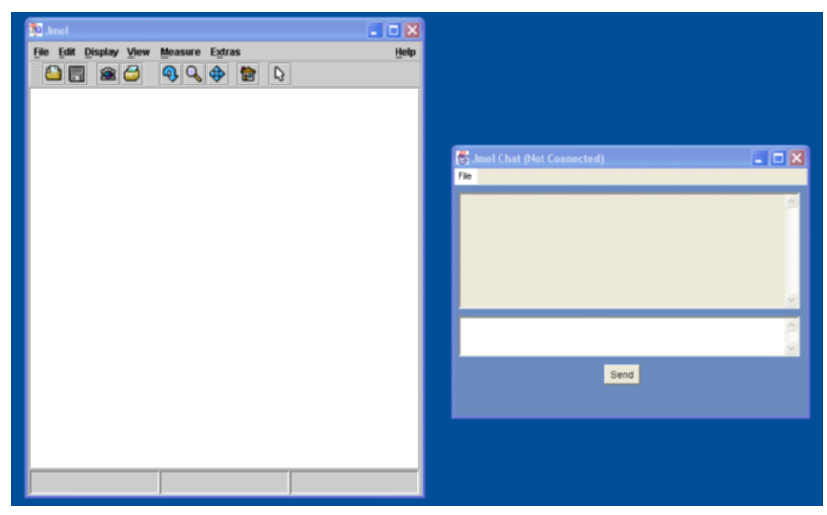

Figure 4 Collaborative Jmol GUI and chat loaded and advertising for a peer.

Vibrate.java displays molecular vibrations as animations. As with the Animate class, it contains controls to specify how a sequnce of frames is displayed. The actionPerformed() method, which controls animation playback, is augmented to send a message for every control action performed.

Measure.java is responsible for measuring angles, distances, and dihedrals between atoms. It also maintains a measurement table containing the various measurements made by the user. It was modified to send messages that correspond to a specific "Action." Actions include measuring or deleting a distance, a bond angle, or dihedral angle.

Jmol.java is the main Java class. It was modified to establish communication channels by implementing theJXTA interfaces: PipeMsgListener, DiscoveryListener, and OutputPipeListener. PipeMsgListener is used for handling incoming messages; DiscoveryListener is used for discovering pipe advertisements; and OutputPipeListener is used when an instance of an OutputPipe is created.

Finally, Jmol was augmented with a chat facility that provided for saving chats and reloading for further review.

\section{Running Collaborative Jmol}

The collaborative version of Jmol is implemented as a Java application. Once Jmol has been loaded from a command line prompt, a dialog box appears. For first time execution, the dialog box sets up user id and security parameters (Figure 2). Subsequent invocations bring up only a login dialog. Once logged in, a command line message requests the user to either create an advertisement for a new peer session, or find an advertisement for an existing peer session (Figure 3). To originate peer collaboration, the former is selected; otherwise the peer will attempt to find the originating peer on the network. If the user has selected advertisement, the Jmol GUI will load and advertise to the netgroup (Figure 4); otherwise Jmol will search 
the network looking for a peer connection. When an advertising peer has been located (Figure 3) the searching peer has the option to make a connection. Once the connection is made, the Jmol GUI will be displayed.

One peer will load a molecule through the "File" dropdown menu to begin collaboration. This filename and a "Draw" message will be sent to the other peer for it to automatically load the molecule from its local directory. Either collaborator can transform the molecule, display its properties, or make measurements. For example, when one collaborator selects rotation, and drags the mouse across the molecular representation, both see exactly the same view, smoothly rotating in three dimensions about the $x$ and $y$-axes. When a collaborator selects measure mode, a table appears that is filled with geometric information as the requisite atoms are selected with the mouse (Figure 5). When the "Add to Measurement List" button is chosen, the measurement is appended to the image at both locations. This text is a three-dimensional graphic of geometric information, and travels along with the molecule as it is rotated, scaled, and translated.

During the course of their engagement, collaborators may carry on a text chat with the built-in chat facility or use any other communication tool available for audio or video conferencing, such as Microsoft's NetMeeting. When it is time to end the session, all that is required is to shut down the application.

\section{Discussion and conclusions}

The goal of this project was to create a collaborative visualization application from a single-user program. Its implementation is consistent with the design objectives put forth by Wood and coworkers [3]. Collaborative visualization systems should allow users to share program control, collaborate dynamically, use the software in an instruction scenario, learn it easily, and exchange data readily. Collaborative Jmol meets these design goals. Either participant may control any of Jmol 's parameters at any time, supporting variability in visualization scenarios. Jmol allows either participant to work as an instructor, because each peer may take control of the analysis at any time, and every action in a sequence is replicated on the collaborating peer. Jmol is easy to learn because the visualization components of the program remain unchanged; only menu-driven networking components have been added. Finally, all data is exchanged between peers, meaning both peers are synchronized in identical collaboration states.

Peer-two-peer bi-directional synchronization is executed in Jmol using two asynchronous pipes by binding each peer to the input end of one pipe and the output end of the other. When a GUI event occurs, the sending peer transmits a keyword designating the GUI operation along with its numerical operands through the input end of the pipe, while the receiving peer extracts the message at the pipe's

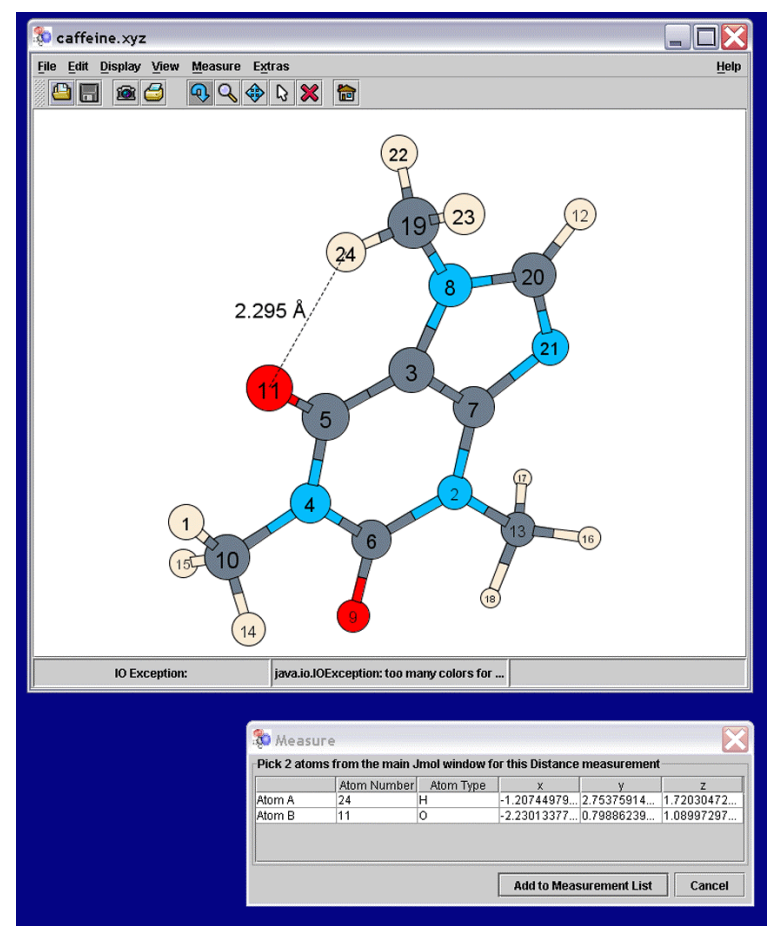

Figure $5 \mathrm{Jmol}$ in measurement mode displaying the caffeine molecule.

output end. Because these pipes act independently, it is possible for both peers to transmit messages simultaneously, possibly corrupting visualizations. Although access to the shared state may be regulated, the current version of Jmol assumes a free access model similar to cAVS, in which any participant may affect the common collaborative state at any time [17]. However, since Jmol will be used as part of a teleconferencing suite, it is expected that such problems will be mitigated because both participants' actions will be coordinated as part of discussion and exploration.

The process of adapting Jmol was constrained in two ways. First, domain experts, not software engineers, created Jmol. As such, these individuals typically are less concerned with a flexible software design that makes for ease of expansion and adaptation, than with building a product to meet current visualization needs. Yet, the modularity of Java's AWT, and its concomitant isolation of interface methods, made it relatively straightforward to embed the requisite JXTA code. Second, JXTA was in early development when this research began, today, version 2 of JXTA is available. Some of its classes were not implemented, unstable, or undergoing change. Therefore, it constrained the adaptation process. Despite this, JXTA's core functionally was sufficiently well developed to adapt Jmol for basic collaboration.

Jmol was selected for adaptation because it represented a useful software product with enough functionality to 
embody significant program structure and complexity. This decision was based on experience using the program, not on an analysis of the source code. It was only after Jmol was selected that its source code was examined. Then the Jmol transformation was achieved by an undergraduate computer science major.

What this research demonstrates is that within a twomonth period, working part-time, an individual with no knowledge of the visualization software, and learning JXTA on the fly, can transform a single-user program into a functional and useful collaborative application. Therefore, it follows that a domain expert, who thoroughly understands the visualization application, could create a collaborative system within a very short time frame.

In conclusion, the demonstrated ability of JXTA to quickly create two person, peer-to-peer collaborative software from single-user visualization systems means that collaboration across the web will become easier to initiate, more spontaneous, and be supported by a wide range of visualization software.

\section{Future Work}

JXTA offers the opportunity to have many peers collaborate, but a design change in Jmol may be required. Presently, there is no visual or auditory cue from Jmol that reveals which peer is controlling the interface. The natural assumption is that the other peer is in control. Additional peers create ambiguity. Without some cue from the software, knowledge of who is in control is uncertain at best. Even if the collaborators employ audio or video conferencing software as part of their session, there is no way of knowing with certainty who is manipulating the model.

This issue is part of a more general problem with which designers of large-scale networked collaborative environments must contend - how to provide a shared sense of presence. CVEs should represent all participants and their actions in the virtual space they share. In Jmol that would mean at the very least the GUI should convey who is currently controlling the software. Hence, the next step in the process of adapting Jmol is to solve this design problem, so the identity of each user in the collaboration is clearly conveyed.

\section{Acknowledgements}

This project was underwritten in part by National Science Foundation Grant ANI-0125043. The author would also like to thank Susan Merritt, Dean of the School of Computer Science and Information Systems, for her continued support.

\section{References}

[1] S. Singhal and M. Zyda. Networked Virtual Environments. Addison-Wesley, 1999.
[2] W. Hibbard, "VisAD: Connecting People to Computations and People to People," Computer Graphics 32,3, 1998, pp. 1012.

[3] J. Wood, H. Wright, K. Brodlie, "Collaborative Visualization," IEEE Visualization '97, 1997, pp. 253-260.

[4] P. Isenhour, J. Begole, W.S. Heagy, and C.A. Shaffer, "Sieve: A Java-Based Collaborative Visualization Environment," IEEE Visualization '97 Late Breaking Hot Topics Proceedings, October 22-24, 1997, pp. 13-16.

[5] A. Pang and C.Wittenbrink, "Collaborative Visualization with CSPRAY," IEEE CG\&A, (March/April) 1997, pp. 32-41.

[6] C. Bajaj and S. Cutchin, "Web based Collaborative Visualization of Distributed and Parallel Simulations," Proceedings of IEEE Parallel Visualization and Graphics Symposium, October 1999, pp. 47-54.

[7] M.Abbott and L.K. Jain, "DOVE: Distributed Objects based scientific Visualization Environment," ACM 1998 Workshop on Java for High-Performance Network Computing. ACM Press, 1998.

[8] “Jmol," http://jmol.sourceforge.net/ (current 23 Mar. 2002).

[9] "Project JXTA," http://wwws.sun.com/software/jxta/ (current 23 Mar. 2003).

[10] D. Brookshier, D. Govoni, N. Krishnan. JXTA: Java P2P Programming. Sams, 2002.

[11] B. Wilson. JXTA. New Rider's Publishing, 2002.

[12] S. Su, R. Loftin, D. Chen, Y. Fang, and Ch. Lin, "Distributed Collaborative Virtual Environment: PaulingWorld." Proceedings of 10th International Conference on Artificial Reality and Telexistence, 2000, pp. 112-117.

[13] C.C. Huang, G.S. Couch, E.F. Pettersen, and T.E. Ferrin, "Chimera: An Extensible Molecular Modeling Application Constructed Using Standard Components," Pacific Symposium on Biocomputing 1, 1996, p. 724.

[14] P.E. Bourne, M. Gribskov, G. Johnson, J. Moreland, and H. Weissig, "A Prototype Molecular Interactive Collaborative Environment (MICE)," Pacific Symposium on Biocomputing, Eds. R Altman, K. Dunker, L. Hunter, and T. Klein, 1998, pp. 118129.

[15] "JMV: Java Molecular Viewer," http://www.ks.uiuc.edu/Research/jmv/(current 23 Mar. 2003).

[16] "Java Mage: Kinemages in your web browser," http://kinemage.biochem.duke.edu/javamage/java.html (current 23 Mar. 2003).

[17] "Collaborative AVS," http://www.tacc.utexas.edu/cavs/overview.html (current 3 May 2003) 